\title{
MicroRNAs and glioblastoma; the stem cell connection
}

\author{
J Godlewski ${ }^{1}$, HB Newton ${ }^{1}$, EA Chiocca ${ }^{1}$ and SE Lawler ${ }^{*, 1}$
}

Recent data draw close parallels between cancer, including glial brain tumors, and the biology of stem and progenitor cells. At the same time, it has become clear that one of the major roles that microRNAs play is in the regulation of stem cell biology, differentiation, and cell 'identity'. For example, microRNAs have been increasingly implicated in the regulation of neural differentiation. Interestingly, initial studies in the incurable brain tumor glioblastoma multiforme strongly suggest that microRNAs involved in neural development play a role in this disease. This encourages the idea that certain miRs allow continued tumor growth through the suppression of differentiation and the maintenance of the stem cell-like properties of tumor cells. These concepts will be explored in this article.

Cell Death and Differentiation (2010) 17, 221-228; doi:10.1038/cdd.2009.71; published online 12 June 2009

Glioblastoma multiforme is the most common and aggressive primary brain tumor, with approximately 10000 new cases per year in the US. ${ }^{1}$ Median survival is 14 months, even with the most up-to-date regimen combining aggressive surgery followed by concomitant radiation and temozolomide chemotherapy. ${ }^{2}$ There is a recognized need for new approaches based on increased understanding of the biological and molecular nature of these tumors. Numerous molecular events have been identified in high-grade gliomas, including EGF receptor amplification and deletion of the tumor suppressors PTEN and $\mathrm{p} 16^{\mathrm{INK} 4 \mathrm{~A}} / \mathrm{p} 14^{\mathrm{ARF}}{ }^{3}$ These alterations facilitate cell survival, proliferation, and migration through activation of specific intracellular pathways including PI3K and MAPK, as well as suppression of Retinoblastoma activity allowing cell cycle progression. In addition to these well-known alterations it is increasingly appreciated that pathways involved in the regulation of development and stem cell behavior such as Notch and Hedgehog are important in glioma biology. ${ }^{4}$ In fact, stem cell biology has found increasing overlap with oncology, in part because of the high profile of the cancer stem cell hypothesis, and also because of the realization that genes important in stem cell self-renewal also play a role in cancer.

Other recent important developments include the discovery of microRNAs, which have a central role in the regulation of gene expression, and are now clearly linked with cancer. ${ }^{5,6}$ MicroRNAs are approximately 23 nucleotide noncoding RNAs that act as important regulators of gene expression by downregulating expression of target genes through regions of partial complementarity in their $3^{\prime}$-UTRs. ${ }^{7}$ At present approximately 1000 microRNAs are thought to exist in the human genome, although the exact number is not yet clear.
Each microRNA may have hundreds of targets, and many genes are targeted by multiple microRNAs, thus leading to potentially highly complex regulatory networks. Details of microRNA processing and regulation have been described in detail elsewhere. ${ }^{5-7}$ Distinct patterns of microRNA expression have been observed in many cancers including glioblastomas, and the functional significance of some of these microRNAs alterations is beginning to emerge. ${ }^{8-11}$ These data indicate that microRNAs play roles in multiple hallmark biological characteristics of glioblastoma, including cell proliferation, invasion, angiogenesis, and also in glioma stem-like cell behavior, which will be the main focus of this article.

\section{Stem Cells and Glioblastoma}

It was initially hypothesized in leukemia and breast cancer that malignancies might include a relatively small percentage of cells that retain stem cell properties and that these cells are the truly tumorigenic subset. ${ }^{12,13}$ Similar subpopulations of cells have subsequently been isolated from additional cancer types, including glioblastoma. ${ }^{14}$ In these studies, patient tumor cells were separated based on the expression of the putative stem cell marker CD133. The CD133 ${ }^{+}$cell population is highly tumorigenic in vivo whereas CD133- ${ }^{-}$cells do not form tumors even at high numbers. $\mathrm{CD} 133^{+}$cells also show increased resistance to radiation and chemotherapy, ${ }^{15}$ and increased angiogenic potential. ${ }^{16}$ These data have led to the hypothesis that glioblastomas are maintained by a relatively small population of cancer stem cells, and that effective therapies must target this treatment resistant subpopulation. However, this hypothesis remains a work in progress. For

\footnotetext{
${ }^{1}$ Dardinger Laboratory for Neuro-oncology and Neurosciences, Department of Neurological Surgery, The Ohio State University Medical Center and James Comprehensive Cancer Center, Columbus, OH 43210, USA

*Corresponding author: SE Lawler, Department of Neurological Surgery, The Ohio State University Medical Center and James Comprehensive Cancer Center, Wiseman Hall, 400 West 12th Avenue, Columbus, OH 43210, USA. Tel: +614 292 3384; Fax: + 614688 4882;

E-mail: sean.lawler@osumc.edu

Keywords: microRNA; glioblastoma; stem cell; development; brain

Abbreviations: PTEN, phosphatase and tensin homolog; MAPK, mitogen activated protein kinase; PI3K, phosphatidyl-inositol-3-kinase; UTR, untranslated region Received 18.3.09; revised 06.5.09; accepted 06.5.09; Edited by G Melino; published online 12.6.09
} 
example, more recent data have identified a novel putative $\mathrm{CD}_{133^{-}}$tumor stem cell population that is tumorigenic. ${ }^{17}$ In addition, assays for cancer stem cells in nude mouse models maybe selecting not for cancer stem cells, but for cancer cells that can thrive in an immunocompromised host. ${ }^{18}$ Recent data show that CD133 is a hypoxia-induced protein, bringing into question its function in stem cell biology. ${ }^{19} \mathrm{~A}$ stem or progenitor cell-like subpopulation may soon be fully characterized in glioblastoma, but more work is needed to clarify this difficult question.

Nonetheless, it is abundantly clear that glioblastoma has many similarities with neural stem cells. Glioblastoma cells can be grown readily under conditions originally designed for the isolation of normal neural stem cells, proliferate indefinitely, self-renew, and can be induced to differentiate along neural, astrocytic, and oligodendroglial lineages, similar to normal neural stem cells. ${ }^{14}$ Glioblastoma cells grown in this manner are also much more genetically stable and similar to their parental tumors than cells grown as monolayers in traditional cell culture, ${ }^{20}$ and tumors established from these cells are more representative of human gliomas in terms of their pathological characteristics. This suggests that maintaining a stem-like cell population in culture allows the more faithful maintenance of in vivo tumorigenicity. Proteins associated with stem and progenitor cells such as Nestin, Bmi-1, Olig2, and Sox2 are also highly expressed in glioblastoma. ${ }^{21}$ Although glioma cells may exploit mechanisms of self-renewal to carryout unlimited proliferation, it is also clear that the mechanisms that allow normal neural stem cells and progenitor cells to fully differentiate are likely to not operate normally in glioblastoma. Understanding how glioblastoma remains in this stem-like, undifferentiated state will allow the development of novel therapies that may promote differentiation or target the stem cell-like properties of glioblastoma. As described in this article, several of the microRNAs so far associated with glioblastoma may normally function to regulate neural stem cell self-renewal and differentiation during development and their dysfunction may contribute to the maintenance of an undifferentiated proliferative phenotype by preventing the expression of targets involved in differentiation and conversely by allowing the expression of targets involved in stem cell renewal.

\section{MicroRNAs in Neural Development}

Although microRNAs have been implicated in an increasing number of biological processes, it is now well recognized that one of the major functions of this class of molecules is in the regulation of development. ${ }^{22}$ Many microRNAs are regulated during development and have tissue-specific expression. Indeed, the first microRNA discovered, lin-4, was identified based on its involvement in the temporal control of postembryonic development. ${ }^{23,24}$ Another microRNA, let-7, was then discovered that is also involved in developmental timing in Caenorhabditis elegans. ${ }^{25}$ Ablation of these microRNAs leads to disruption of development at different larval stages. Lin-4 functions in early development through targeting lin-14 and lin28 genes, whereas let-7 regulates later development through the regulation of lin-41 among others (reviewed in Ref. ${ }^{22}$ ). Increasing examples of microRNA effects in mammalian development are emerging. For example, miR-196 acts upstream of HOXB8 and Sonic hedgehog in limb development. ${ }^{26}$ miR-1 and miR-133 are important in muscle development, ${ }^{27}$ and miR-181 is necessary to establish B-lymphoid cell identity in mice. ${ }^{28}$ An important theme is the emerging role of microRNAs in the regulation of stem cell self-renewal and differentiation. ${ }^{29}$ Ablation of Dicer leads to defects in embryonic stem (ES) cell division and proliferation, ${ }^{30}$ causing death in mice and a complete loss of pluripotent stem cells. ${ }^{31}$ Dicer ablated ES cells also fail to differentiate; thus microRNAs can affect stem cell behavior at the levels of both proliferation and differentiation. Several stem cell-specific microRNAs have been identified, whose expression is reduced on ES cell differentiation. ${ }^{32}$ The specific roles of these microRNAs are beginning to be elucidated. A recent study showed that microRNAs could influence differentiation of ES cells through target sites in the coding sequences of various genes associated with stem cells. $^{33}$ It has been reported that the degree of cellular differentiation correlates with a more complex microRNA signature,${ }^{34}$ and that microRNA profiles in stem cells resemble those seen in cancer cells. ${ }^{35}$

Increasing evidence strongly supports a key role for microRNAs in neural development (reviewed in Ref. ${ }^{35-37}$ ). The exquisite regulation of neural stem cells gives rise to the glial and neural progenitor cells that ultimately form the fully differentiated repertoire of glia and neurons that constitute the bulk of the central nervous system. Neural stem cells in the adult brain reside largely in the subventricular zone and in the dentate gyrus. They have the capacity to self-renew and differentiate into both neural and glial lineages. Seventy percent of known microRNAs are expressed in the brain, many of which seem to be exclusively expressed in the CNS. ${ }^{38,39}$ The most highly expressed microRNAs in brain are miR-124 and miR-128, both of which are preferentially expressed in neurons. Other microRNAs with a high degree of brain specificity include miR-101, -127, -131, and -132 . $^{39}$ The importance of microRNA in neural development has been highlighted by the effects of ablation of the microRNA processing machinery. This revealed altered brain morphogenesis in zebrafish, ${ }^{40}$ and the absence of Dicer from dopaminergic neurons in mice leads to their progressive loss. ${ }^{41}$ Loss of Purkinje cells of the cerebellum, ${ }^{42}$ and neo-cortical neurons has also been described ${ }^{43}$ indicating the importance of proper microRNA processing in brain development.

The relationship of microRNAs and stem cells makes it tempting to speculate that microRNAs may function in cancer by subverting normal stem cell functions. For example, it has been suggested that microRNAs are necessary for proper stem cell division allowing stem cells to override the G1/S checkpoint to sustain continual division over a long period of time. ${ }^{44}$ Cancer cells may exploit these mechanisms to bypass the G1/S checkpoint, as described for miR-124 and miR-137, which are very weakly expressed in glioma cells allowing high expression of CDK6. ${ }^{11}$ Recent research indicates that microRNAs have an important role in regulating stem cell self-renewal and differentiation by repressing the translation of target mRNAs in stem cells and their differentiating daughter cells. ${ }^{45,46}$ The expression of microRNA after differentiation of neural stem cells corresponds with cell lineage. MiR-124 and miR-128 are specific to the neural 
lineage, whereas miR-26, miR-29, and miR-23 are expressed preferentially in astrocytic lineages. ${ }^{39,47}$ The most commonly identified microRNAs in neural precursor cells identified by sequencing are let-7 family members followed by miR-181a/b, miR-17-20, miR-130/301, miR-21, miR15/16, miR-9, and miR-26. ${ }^{48}$ Thus, microRNAs play important roles in development, and may also strongly influence the behavior of neural stem and progenitor cells - these are the normal cell types thought to be most similar biologically to glial tumor cells. ${ }^{21,49}$

\section{MicroRNAs in Glioblastoma}

Similar to other cancers, a characteristic microRNA expression pattern is seen in glioblastoma. ${ }^{8-11}$ These Studies reveal a characteristic pattern of microRNAs whose expression differs from surrounding normal brain tissue. These studies consistently show that miR-21 has the highest expression levels in glioblastoma and several microRNAs are weakly expressed compared with normal brain, including miR-124, miR-7, and miR-128. Interestingly, these microRNAs are also implicated in neuronal differentiation as described in this article. For the most part, little is yet known about the mechanisms by which microRNAs are altered in glioblastoma, but all the usual genomic regulatory systems are likely to operate (methylation, mutations, gene deletion, and amplification) as well as transcriptional and microRNA-specific mechanisms, such as altered processing, editing, and mutation of sequences in complementary target-binding sites.

The use of profiling to identify microRNA alterations presents some technical issues - this is particularly pertinent in brain when comparing tumor cells, which seem to resemble glial progenitor cells, with normal tissue, particularly if that tissue is largely neuronal in composition. For example, the identification of miR-124 and miR-128 as 'downregulated' in gliomas may be in part because of their high expression in neurons. However, because functional studies have shown that overexpression of each of these microRNAs in glioma slowed proliferation and targeted relevant genes, these alterations are clearly of biological importance. Silber et al., (Ref. ${ }^{11}$ ) used a Taqman-based RT-PCR approach to identify altered microRNAs, and compared grade III anaplastic astrocytomas, glioblastoma, and glioses from epilepsy patients. The use of RT-PCR gives a highly sensitive, specific, and quantitative readout, and requires very low sample amounts, but is limited in the detection of microRNAs with $3^{\prime}$ alterations. The other studies carried out so far used microarray chips with glioblastoma samples, and adjacent normal tissue, ${ }^{8-10}$ as well as cell lines, ${ }^{9,10}$ low passage glioblastoma cells, and non-neoplastic fetal brain. ${ }^{9}$ Chips offer the advantage of high microRNA coverage (474 human microRNAs ${ }^{10}$ ), and potentially very high sample throughput. More RNA is required (low micrograms) than PCR, and certain probes may suffer from lack of specificity because of the similarity of some microRNAs. Despite these differences in methodology, the conclusions drawn in each study reveal a group of consistently altered microRNAs. In order to identify further relevant microRNAs in glioblastoma, comparisons of glioma stem cell lines with normal adult human neural stem cell lines will be highly relevant. The recently published Cancer Genome Atlas dataset contains information on
microRNA expression and prognosis, as well as deletions, amplifications, and methylation events that will become an important resource for establishing the importance of specific microRNAs in glioblastoma. The emergence of high-throughput sequencing technology will also assist in the identification of microRNA alterations, and has the ability to identify editing events and novel microRNAs. MicroRNA profiling studies on other CNS tumors are still at an early stage. A recent report described microRNA alterations in medulloblastoma determined by RT-PCR of 248 microRNAs in 14 medulloblastomas compared with 7 adult and fetal normal cerebellar controls. ${ }^{50}$ This showed some similarities with gliomas, with miR-21 upregulated, and downregulation of miR-124, miR-128, and miR-7 among many others. Interestingly, miR-137, which is downregulated in glioblastoma is upregulated in meduloblastoma samples, for unknown reasons. Another study showed miR-199b-5p downregulation which was associated with metastatic spread in medulloblastomas, and affects stem cell signaling through inhibition of the Notch target Hes $1 .{ }^{51}$

MicroRNAs have now been shown to affect the expression of numerous genes involved in cancer pathways. Figure 1 shows a diagram of the PI3 kinase pathway, one of the key regulators of tumorigenesis in glioblastoma and in other cancers. This is overlaid with microRNAs that have been shown to target these molecules. It can be appreciated that microRNAs affect complex gene networks, and that they play very important roles in this key pro-tumorigenic pathway. However, it has quickly become apparent that the role of microRNAs in cancer is more complex than simply acting as regulators of known oncogenes and tumor suppressor genes. This is illustrated by the striking correlation between microRNA alterations in glioblastoma and those so far identified in neural development (summarized in Table 1). MiR-124 and miR-7 have been shown to play roles in promoting neural development and are highly expressed in neurons and not in

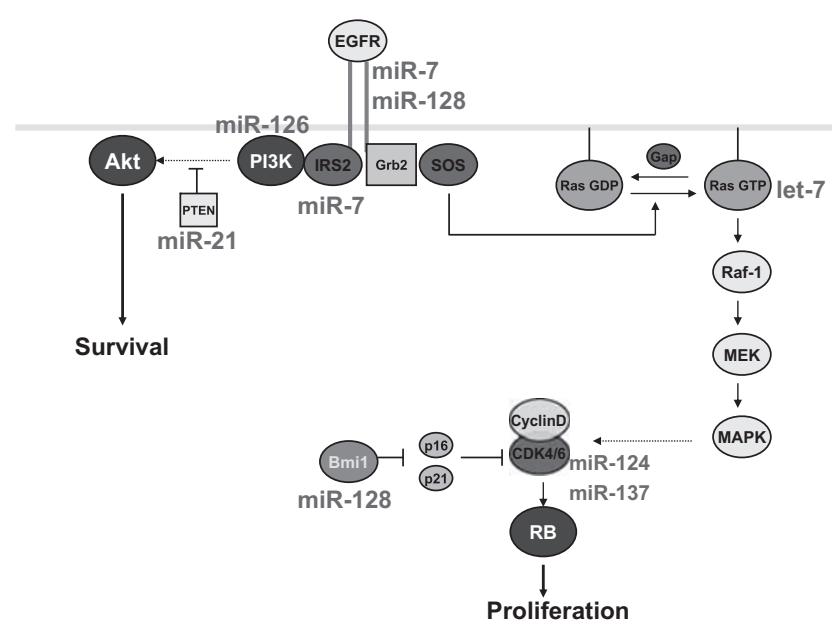

Figure 1 The interface of microRNAs with the known oncogenic EGFR signaling pathway. MicroRNAs that have been shown to target each gene experimentally are shown next to their target genes. This is a composite of several cancer types, and the targeting of PTEN by miR-21 and PI3K by miR-126 has yet to be demonstrated in glioblastoma. This network is likely to become a great deal more complex in the coming years as more microRNA/target pairs are validated 
Table 1 Validated glioblastoma microRNAs and their functions in neural development and known targets

\begin{tabular}{|c|c|c|c|}
\hline MicroRNA & Normal biology & Glioblastoma biology & Targets \\
\hline $\mathrm{miR}-124$ & $\begin{array}{l}\text { Promotes neural differentiation } \\
\text { Expressed in differentiating and mature neurons - } \\
\text { not precursors } \\
\text { Repressed by REST } \\
\text { Suppresses nonneuronal genes } \\
\text { Highly expressed in brain (neurons) }\end{array}$ & $\begin{array}{l}\text { Weakly expressed in gliomas } \\
\text { Overexpression slows proliferation } \\
\text { Overexpression promotes neural differentiation }\end{array}$ & $\begin{array}{l}\text { ITGB1 } \\
\text { LAMC1 } \\
\text { CDK6 } \\
\text { SCP1 } \\
\text { PTBP1 }\end{array}$ \\
\hline MiR-137 & Not known & $\begin{array}{l}\text { Weakly expressed in gliomas } \\
\text { Suppressed by methylation } \\
\text { Overexpression slows proliferation } \\
\text { Overexpression promotes neural differentiation }\end{array}$ & CDK6 \\
\hline MiR-128 & $\begin{array}{l}\text { Not known } \\
\text { Highly expressed in brain (neurons) }\end{array}$ & $\begin{array}{l}\text { Weakly expressed in gliomas } \\
\text { Overexpression slows proliferation } \\
\text { Overexpression blocks glioma stem cell } \\
\text { self-renewal }\end{array}$ & $\begin{array}{l}\text { Bmi1 } \\
\text { E2F3A } \\
\text { EGFR }\end{array}$ \\
\hline MiR-7 & $\begin{array}{l}\text { Promotes neural differentiation (Drosophila retina) } \\
\text { Promotes muscle differentiation (mammalian) } \\
\text { Represses transcription factors to promote } \\
\text { differentiation } \\
\text { Expressed in brain }\end{array}$ & $\begin{array}{l}\text { Weakly expressed in gliomas } \\
\text { Processing impaired } \\
\text { Overexpression slows proliferation and } \\
\text { migration }\end{array}$ & $\begin{array}{l}\text { EGFR } \\
\text { IRS2 } \\
\text { Yan (Drosophila) } \\
\text { PAK1 }\end{array}$ \\
\hline MiR-9/9* & $\begin{array}{l}\text { Neural lineage differentiation from embryonic stem } \\
\text { cells (mammalian) } \\
\text { Inhibits neuronal fate (Drosophila) } \\
\text { Specifically expressed in brain }\end{array}$ & $\begin{array}{l}\text { Expressed in gliomas } \\
\text { Functions not known }\end{array}$ & $\begin{array}{l}\text { REST } \\
\text { Co-REST }\end{array}$ \\
\hline
\end{tabular}

neural progenitor cells. They are also very weakly expressed in glioblastoma, and have profound effects on glioma cells when ectopically expressed. Similarly, miR-128 is weakly expressed in glioblastoma and highly expressed in neurons, although its function is not yet clear, but it may well control stem cell self-renewal in both tumor and normal cells. These initial data encourage the idea that certain microRNAs allow continued tumor growth through the suppression of differentiation and the maintenance of the stem cell-like properties of tumor cells as discussed below.

MicroRNA-124. There are three miR-124 genes in humans with identical mature microRNA sequences (these are referred to as a, b, and c, or in miRbase as 124-1, 124-2, and 124-3). MiR-124 is $100 \%$ conserved at the nucleotide level from worms to humans and is the most abundant microRNA in the brain, accounting for at least $25 \%$ of total brain microRNA. ${ }^{37}$ In the mouse, miR-124 is mostly expressed in differentiating and mature neurons, compared with neural progenitors, and its expression correlates with the first appearance of differentiated neurons in culture from neural progenitor cells. ${ }^{47,52}$ MiR-124 plays an important functional role in promoting neural differentiation by repressing a large number of non-neural genes. ${ }^{52}$ The expression of miR-124 is controlled by the REST (RE1 transcription factor) repressor of transcription that inhibits miR-124 expression in both non-neural cells and progenitors, but is absent in mature neurons thus allowing miR-124 expression. REST is a key transcriptional repressor that functions in neuronal development by targeting many neuronal genes in addition to miR-124, and represses the expression of the neural transcripts in non-neuronal cells.
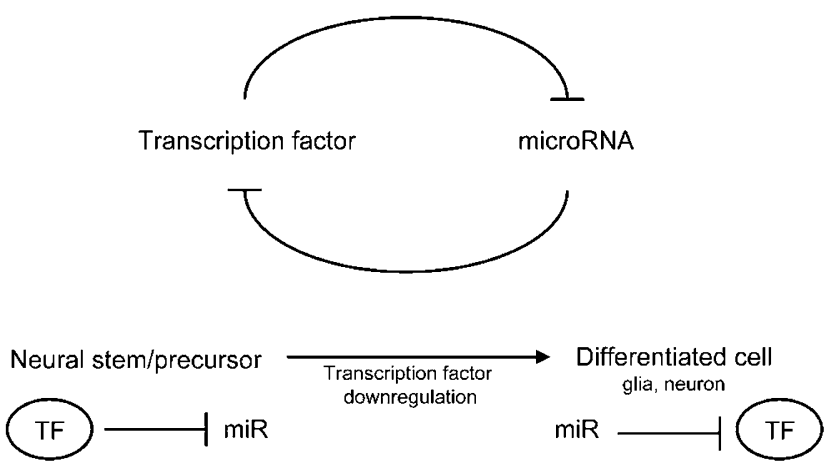

Figure 2 Reciprocal negative feedback circuitry in cell differentiation. Several examples have now been reported of reciprocal or double-negative feedback circuits involved in the regulation of differentiation by microRNAs. These include miR-7/Yan (Drosophila photoreceptor), miR-29/YY1 in muscle differentiation and miR-124/SCP1. In this example, a transcription factor represses a microRNA in a stem/precursor cell. Lowering of transcription factor levels (by degradation or other means) allows microRNA expression, which then further represses transcription factor expression, helping to commit the cell toward a fully differentiated state. It is highly likely that this circuitry is disrupted in cancer

REST expression is higher in pluripotent stem cells, lower in adult neural stem cells, and minimal in postmitotic neurons. ${ }^{53}$ Thus, neural differentiation may involve the inactivation of REST followed by downregulation of non-neural genes by miR-124. Some important targets of miR-124 have been identified in neural differentiation - for example, downregulation of the RNA-binding protein PTBP1 by miR124 during neural differentiation promotes a neuron-specific splicing pattern. ${ }^{54}$ Other miR-124 targets such as SCP1, laminin $\gamma 1$, and integrin $\beta 1$ are downregulated in 
differentiating neurons. ${ }^{55}$ SCP1 is an antineural factor that acts as a part of the REST complex to prevent the expression of neural genes in nonneural tissues. ${ }^{56}$ This results in the establishment of a double-negative feedback loop between miR-124 and the REST complex. Thus, microRNA-124regulated circuitry is emerging in which it plays a central role in neural differentiation. This is a common theme in microRNA-induced cellular differentiation. The basic principle is shown in Figure 2.

The expression levels of miR-124 are significantly decreased in anaplastic astrocytomas and glioblastoma relative to non-neoplastic brain tissue. ${ }^{11}$ Indeed, miR-124 seems to be the most differentially expressed microRNAs in glioblastoma compared with normal brain. MiR-137 is also weakly expressed in glioblastoma but its function in normal neural development is not known. When cultured early passage subventricular zone mouse neural stem cells were deprived of growth factors, a steady increase in the number of Tuj-positive neuroblasts was accompanied by increased miR-124 and miR-137 expression, by 8- and 20-fold, respectively. ${ }^{11}$ Importantly, human $\mathrm{CD}_{133^{+}}$glioma-derived stem cells, when overexpressing miR-124 and miR-137, displayed a dramatic increase in Tuj-positive cells and a concurrent decrease in GFAP-positive cell numbers. In addition, transfection with miR-124 or miR-137 induced morphological changes and marker expression consistent with neural differentiation in mouse neural stem cells as well as in oligodendrogliomaderived stem cells. These alterations in differentiation were accompanied by reduced self-renewal and tumorigenicity. Moreover, miR-124 and miR-137 induced G1 cell-cycle arrest in U251 and SF6969 glioblastoma cells, which was associated with decreased expression of CDK6 and phosphorylated
Retinoblastoma protein. Consequently, CDK6 was demonstrated to be the direct target of both microRNAs. The expression of miR-137 was increased several-fold in glioma cell lines after treatment with demethylating agents, suggesting that its suppression plays an important role in glioma biology. These results indicate miR-124 and miR-137 are potent antiproliferative and pro-differentiating factors with tumor-suppressor activity in glioma, and may therefore be of therapeutic relevance. The roles of miR-124 along with miR128 and miR-7 are illustrated in Figure 3.

MicroRNA-128. MiR-128 is encoded by two distinct genes (miR-128-1 and miR-128-2), which encode identical mature sequences. miR-128 was significantly downregulated when glioblastoma samples were compared with adjacent, nonpathological tissue from the same patients. ${ }^{10}$ The introduction of miR-128 into glioma cells caused a marked reduction in their proliferation in vitro and in vivo. Of several potential target genes examined, the known oncogene and stem cell renewal factor, Bmi1, was demonstrated to be the most consistently downregulated target in a panel of glioma cell lines. In glioma-derived stem cells stably overexpressing miR-128, a large reduction in neurosphere volume and frequency was observed in a self-renewal assay, consistent with Bmi1 loss. Moreover, miR-128-mediated knockdown of Bmi1 enhanced expression of correlative markers including the tumor suppressor p21. Finally, a negative correlation between miR-128 and Bmi1 was demonstrated in glioblastoma samples and adjacent, nonpathological tissue from the same patients by means of RT-PCR and immunohistochemistry. Bmi1 is a polycomb family transcriptional repressor required for postnatal maintenance a

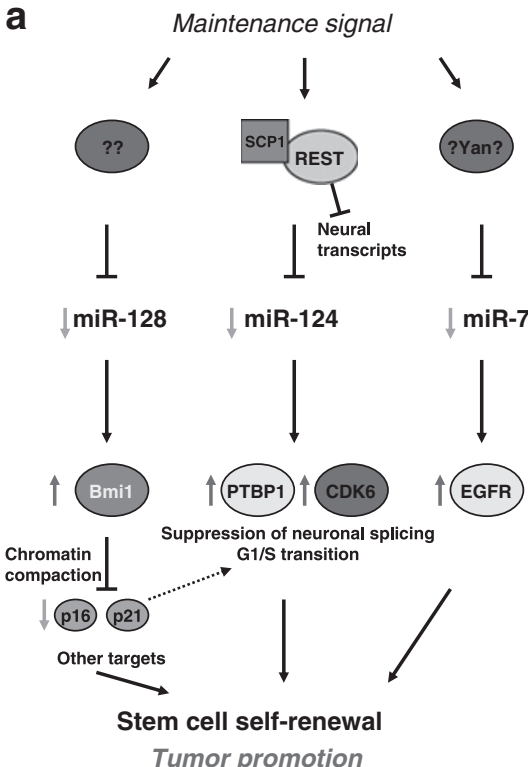

b
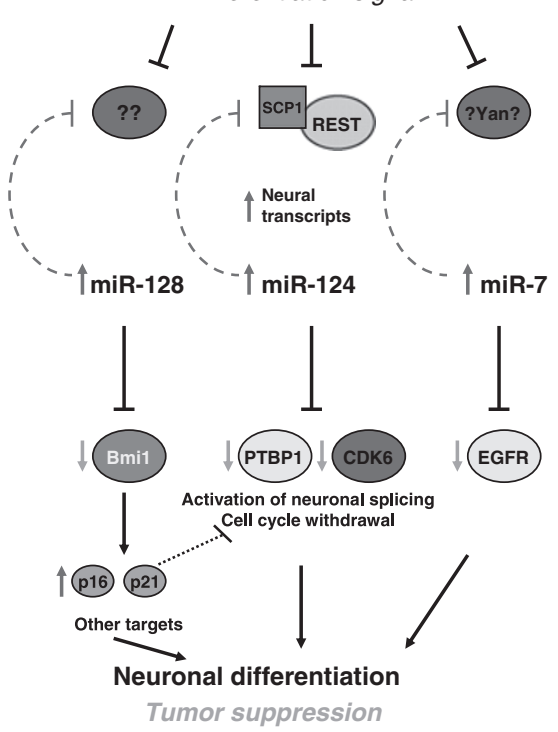

Figure 3 MicroRNA circuitry in neural stem cells. The pathways involved in the regulation of microRNAs involved in neural stem cell biology, which have also been implicated in glioma are shown. (a) Shows the scenario under conditions that favor stem cell maintenance (or tumor growth). (b) Shows the switch that occurs on neuronal differentiation. The release of transcriptional repression of microRNAs initiates self-sustaining double-negative feedback loops, in which increasing microRNA levels lead to further suppression of the upstream inhibitors, and therefore sustained microRNA expression. This is done by REST in the case of miR-124, a putative human homologue of Drosophila Yan (miR-7, although this link is not established in humans as yet), and a putative repressor of miR-128 
of neural stem cells in the peripheral and central nervous system. ${ }^{57}$ Mice lacking Bmi1 suffer from a postnatal selfrenewal defect that leads to depletion of stem cells by early adulthood. (reviewed in Ref. ${ }^{58}$ ). Bmi1 likely functions in the repression of many genes including the potent tumor suppressor $\mathrm{p} 16^{\mathrm{INK} 4 \mathrm{~A}} / \mathrm{p} 14^{\mathrm{ARF}}$ locus. ${ }^{59}$ Thus, in concert with other components including transcription factors, other polycomb proteins and modifiers of histones, Bmi1 maintains neural stem cells in an undifferentiated selfrenewing state. Recently, it was shown that in neural precursors polycomb proteins repress differentiationassociated microRNAs including miR-124, miR-9, miR-153, and miR-137. ${ }^{48}$ Similarly, miR-128 has been shown to be enriched in brain, specifically in neurons, especially in the cortex and hippocampus in the mouse, ${ }^{39}$ but its function in neural differentiation is not known. These glioma studies suggest that the regulation of Bmi1 by miR-128 may be relevant to normal stem cell regulation. Another report on miR-128 in glioma confirmed its downregulation in tumor samples as well as a striking antiproliferative effect, identifying E2F3a as a target. ${ }^{60}$

Interestingly, a recent report described targeting of another polycomb-associated protein, EZH2, in cancer by miR-101. ${ }^{61}$ miR-101 is commonly deleted in cancers, and the finding of another target involved in chromatin modification suggests that this is an important mechanism of microRNA action. The role of miR-101 in glioma is yet to be determined.

MicroRNA-7. Similar to miR-124 and miR-128, miR-7 is also weakly expressed in neural stem cells and is expressed on differentiation in certain neural cell types. MiR-7 is moderately expressed in human brain with only scattered expression in other organs. ${ }^{62}$ In Drosophila miR-7 increases the production of sensory organ precursor cells through actions on Notch target genes such as Enhancer of Split complex and the Bearded complex. ${ }^{63}$ Ectopic miR-7 expression promotes Drosophila photoreceptor neuron differentiation. ${ }^{64}$ MiR-7 is expressed weakly in progenitor cells in the retina, in which its expression is repressed by the Ets domain transcription repressor Yan stimulated by EGFR activation. On differentiation Yan is degraded, thus allowing miR-7 expression. MiR-7 then further increases and maintains its own expression by targeting sequences in the 3'UTR of Yan - thus, forming a reciprocal negative feedback loop. ${ }^{64}$ A similar loop exists between miR-133b and the transcription factor PITX3 during the maturation of midbrain dopamine neurons ${ }^{41}$ suggesting a common mode of regulation. The first microRNA to be shown to be involved in neural development was $C$. elegans Lsy-6, which is involved in a complex negative feedback loop in the specification of specific chemoreceptor neurons. ${ }^{65}$

These microRNA-mediated feedback and feedforward loops are recurrent network motifs. ${ }^{66}$ Circuits in which a microRNA and a target are both regulated by the same upstream factor can lead to either negative feedback or feedforward loops, and may constitute a buffering or stabilization system that enhances the robustness of gene expression. Indeed, miR-7 has recently been implicated in buffering developmental programs against temperature fluctuations in Drosophila. ${ }^{67}$ Circuits in which a microRNA and its target are regulated in the opposite way to a stimulus are strongly implicated in differentiation. It has been suggested that a key function of microRNAs is to reinforce the gene expression profile of differentiated states (as seen for miR124). The dysregulation of this circuitry could enhance transformation, by deregulating gene expression and blocking normal differentiation programs.

MiR-7 was recently shown to be particularly weakly expressed in human glioma specimens. ${ }^{68}$ It was also shown that miR-7 directly targets EGFR through three sites within its $3^{\prime}$-UTR and suppresses Akt activity. Its overexpression in glioma cell lines caused significant decrease in their viability and invasiveness. The effects of miR-7 on glioma stem cell-like cultures have yet to be reported, and its functions in human neurogenesis need further study.

MicroRNA-21. MiR-21 is one of the most commonly upregulated microRNAs in human carcinomas. Its elevated expression was consistently reported in most types of cancer tested and was first reported in glioblastoma. ${ }^{9}$ In this study, significant overexpression of miR-21 in glioma patient tissue samples, early-passage glioma cultures, and established glioma cell lines was shown when compared with nonneoplastic brain tissue or normal human astrocytes. Inhibition of miR-21 led to significantly decreased cell number in several glioma-derived cell lines because of increased apoptosis in vitro ${ }^{9}$ and in vivo. ${ }^{69}$ MiR-21 targets multiple important components of the p53, TGF- $\beta$, and mitochondrial apoptosis tumor-suppressive pathways in glioblastoma. Downregulation of miR-21 in glioblastoma cells caused de-repression of these pathways, causing repression of growth, increased apoptosis, and cell-cycle arrest. ${ }^{70}$ Several well-known glioma tumor-suppressor genes like APAF1 or STAT3, which are predicted miR-21 targets, were shown by microarray to be negatively regulated (reviewed in Ref. ${ }^{71}$ ).

Conflicting results have been published regarding the putative role of miR-21 in stem cell biology. It was reported that the neural repressor REST maintains self-renewal and pluripotency in mouse ES cells through suppression of miR$21 ;{ }^{72}$ however, in another report miR-21 was expressed at similar levels in REST-deficient and wild-type ES cells. ${ }^{73} \mathrm{MiR}$ 21 also targets known stem cell regulators, Nanog and SOX2, in mouse ES cells, which are necessary for stem cell selfrenewal. ${ }^{72}$ Thus, increasing evidence implicates miR-21 in stem cell biology. Whether this relates to tumorigenesis is unclear at present. Its effects on glioma stem-like cell cultures have not been investigated.

MicroRNA-9. Other microRNA candidates with a potential involvement in neural development and glioblastoma include miR-9, although this is less well studied than the examples above. MiR-9 is expressed almost exclusively in the brain and is a mediator of neurogenesis. ${ }^{47,74}$ It supports the proper differentiation of Cajal-Retzius cells in the medial pallium, at least in part through downregulation of Foxg1. ${ }^{75}$ miR-9 directly targets REST and the opposing strand miR-9* targets co-REST, thus promoting neural differentiation. Moreover, miR-9 maybe repressed by REST, thus providing another example of a double-negative or reciprocal feedback loop 
between a microRNA and a regulator of transcription. ${ }^{74} \mathrm{miR}$ 9 is also highly expressed in fetal brain and oligodendroglioma compared with adult brain and normal astrocytes. MiR-9 is overexpressed in glioblastoma and may differentiate primary from metastatic tumors. ${ }^{76}$ This differs from the earlier examples that express low amounts of microRNAs that support neuronal differentiation. The reasons for this are as not clear, and the phenotype of glioma cells with altered miR-9 expression is not yet known.

Finally, additional microRNAs, such as miR-133, miR-125, and miR-388 have been implicated in neuronal differentiation, and may also be downregulated in glioblastoma. These may also contribute to glioma growth through effects on stem cell behavior.

\section{Concluding Remarks}

Several microRNAs have been identified with functional importance in neural development. In parallel, through differential profiling studies microRNAs have been identified with potential importance in glioma biology. There is a remarkable co-incidence in the microRNAs studied in these fields thus far. This may identify potential therapeutic microRNAs, identify novel mechanisms relevant to both tumor biology and neurogenesis, and most certainly supports the concept that gliomas bear a close similarity to normal neural stem cells. Whether this indicates that gliomas arise from neural stem cells is open to debate. MicroRNAs have been implicated in cancer hallmarks including proliferation, reduced apoptosis, invasion, and angiogenesis, but the coincident roles of microRNAs in neural stem cells and glioma strongly suggest a link between these two types of cell. In particular, miR-7 and miR-124 play roles in neural differentiation and are not readily detected in glioma. miR-128 plays a role in glioma, but its function in normal cells is not yet known. Evidence from glioblastoma indicates that ablation of miR-128 may have severe effects because of impairment of stem cell selfrenewal. Importantly, these microRNAs also have pronounced effects on glioma cell growth and migration. Thus, a theme is emerging in which microRNAs that are altered in glioblastoma also play roles in the development of the central nervous system, likely reflecting the similarity between glioblastoma and neural progenitor cell biology. The absence of these microRNAs favors stem cell renewal/proliferation and their presence favors differentiation; thus, it may be possible to harness these activities for the treatment of gliomas and other tumor types. These observations provide important concepts to develop strategies designed to target glioma stem cells in patients.

Acknowledgements. The authors acknowledge the National Institutes of Health, the Esther L Dardinger Endowment for Neuro-oncology and Neurosciences, and The Thomas Jeffrey Hayden Foundation for their support.

1. Davis FG, McCarthy BJ. Current epidemiological trends and surveillance issues in brain tumors. Expert Rev Anticancer Ther 2001; 1: 395-401.

2. Stupp R, Mason WP, van den Bent MJ, Weller M, Fisher B, Taphoorn MJ et al. Radiotherapy plus concomitant and adjuvant temozolomide for glioblastoma. N Engl J Med 2005; 352: 987-996.
3. Furnari FB, Fenton T, Bachoo RM, Mukasa A, Stommel JM, Stegh A et al. Malignant astrocytic glioma: genetics, biology, and paths to treatment. Genes Dev 2007; 21: 26832710

4. Dell'Albani P. Stem cell markers in gliomas. Neurochem Res 2008; 33: 2407-2415.

5. Volinia S, Calin GA, Liu CG, Ambs S, Cimmino A, Petrocca F et al. A microRNA expression signature of human solid tumors defines cancer gene targets. Proc Natl Acad Sci USA 2006; 103: 2257-2261.

6. Esquela-Kerscher A, Slack FJ. Oncomirs - microRNAs with a role in cancer. Nat Rev Cancer 2006; 6: 259-269.

7. Bartel DP. MicroRNAs: target recognition and regulatory functions. Cell 2009; 136 : 215-233

8. Ciafrè SA, Galardi S, Mangiola A, Ferracin M, Liu CG, Sabatino G et al. Extensive modulation of a set of microRNAs in primary glioblastoma. Biochem Biophys Res Commun 2005; 334: 1351-1358.

9. Chan JA, Krichevsky AM, Kosik KS. MicroRNA-21 is an antiapoptotic factor in human glioblastoma cells. Cancer Res 2005; 65: 6029-6033.

10. Godlewski J, Nowicki MO, Bronisz A, Williams S, Otsuki A, Nuovo G et al. Targeting of the Bmi-1 oncogene/stem cell renewal factor by microRNA-128 inhibits glioma proliferation and self-renewal. Cancer Res 2008; 68: 9125-9130.

11. Silber J, Lim DA, Petritsch C, Persson Al, Maunakea AK, Yu M et al. miR-124 and miR-137 inhibit proliferation of glioblastoma multiforme cells and induce differentiation of brain tumor stem cells. BMC Med 2008; 6: 14-22.

12. Huntly BJP, Gilliland DG. Leukemia stem cells and the evolution of cancer-stem-cell research. Nat Rev Cancer 2005; 5: 311-321.

13. Stingl J, Caldas $\mathrm{C}$. Molecular heterogeneity of breast carcinomas and the cancer stem cell hypothesis. Nat Rev Cancer 2007; 445: 111-115.

14. Singh SK, Hawkins C, Clarke ID, Squire JA, Bayani J, Hide T et al. Identification of human brain tumour initiating cells. Nature 2004; 432: 396-401.

15. Bao S, Wu Q, McLendon RE, Hao Y, Shi Q, Hjelmeland AB et al. Glioma stem cells promote radioresistance by preferential activation of the DNA damage response. Nature 2006; 444: 756-760.

16. Bao S, Wu Q, Sathornsumetee S, Hao Y, Li Z, Hjelmeland AB et al. Stem cell-like glioma cells promote tumor angiogenesis through vascular endothelial growth factor. Cancer Res 2006; 66: 7843-7848.

17. Beier D, Hau P, Proescholdt M, Lohmeier A, Wischhusen J, Oefner PJ et al. CD133(+) and CD133(-) glioblastoma-derived cancer stem cells show differential growth characteristics and molecular profiles. Cancer Res 2007; 67: 4010-4015.

18. Quintana E, Shackleton M, Sabel MS, Fullen DR, Johnson TM, Morrison SJ. Efficient tumor formation by single human melanoma cells. Nature 2008; 456: 593-598.

19. Griguer CE, Oliva CR, Gobin E, Marcorelles P, Benos DJ, Lancaster Jr JR et al. CD133 is a marker of bioenergetic stress in human glioma. PLOS ONE 2008; 3: e3655.

20. Lee J, Kotliarova S, Kotliarov Y, Li A, Su Q, Donin NM et al. Tumor stem cells derived from glioblastomas cultured in bFGF and EGF more closely mirror the phenotype and genotype of primary tumors than do serum-cultured cell lines. Cancer Cell 2006; 9: 391-403.

21. Stiles CD, Rowitch DH. Glioma stem cells: a midterm exam. Neuron 2008; 58: 832-846.

22. Wienholds E, Plasterk RH. MicroRNA function in animal development. FEBS Lett 2005; 579: 5911-5922.

23. Lee RC, Feinbaum RL, Ambros V. The $C$. elegans heterochronic gene lin-4 encodes small RNAs with antisense complementarity to lin-14. Cell 1993; 75: 843-854.

24. Wightman B, Ha I, Ruvkun G. Posttranscriptional regulation of the heterochronic gene lin14 by lin-4 mediates temporal pattern formation in C. elegans. Cell 1993; 75: 855-862.

25. Reinhart BJ, Slack FJ, Basson M, Pasquinelli AE, Bettinger JC, Rougvie AE et al. The 21nucleotide let-7 RNA regulates developmental timing in Caenorhabditis elegans. Nature 2000; 403: 901-906.

26. Hornstein E, Mansfield JH, Yekta S, Hu JK, Harfe BD, McManus MT et al. The microRNA miR-196 acts upstream of Hoxb8 and Shh in limb development. Nature 2005; 438: $671-$ 674.

27. Chen JF, Mandel EM, Thomson JM, Wu Q, Callis TE, Hammond SM et al. The role of microRNA-1 and microRNA-133 in skeletal muscle proliferation and differentiation. Nat Genet 2006; 38: 228-233.

28. Chen CZ, Li L, Lodish HF, Bartel DP. MicroRNAs modulate hematopoietic lineage differentiation. Science 2004; 303: 83-86.

29. Hatfield S, Ruohola-Baker H. MicroRNA and stem cell function. Cell Tissue Res 2008; 331: $57-66$.

30. Murchison EP, Partridge JF, Tam OH, Cheloufi S, Hannon GJ. Characterization of Dicerdeficient murine embryonic stem cells. Proc Natl Acad Sci USA 2005; 102: 12135-12140.

31. Bernstein E, Kim SY, Carmell MA, Murchison EP, Alcorn H, Li MZ et al. Dicer is essential for mouse development. Nat Genet 2003; 35: 215-217.

32. Houbaviy HB, Murray MF, Sharp PA. Embryonic stem cell-specific microRNAs. Dev Cell 2003; 5: 351-358.

33. Tay Y, Zhang J, Thomson AM, Lim B, Rigoutsos I. MicroRNAs to Nanog, Oct4 and Sox2 coding regions modulate embryonic stem cell differentiation. Nature 2008; 455 : 1124-1128.

34. Chen C, Ridzon D, Lee CT, Blake J, Sun Y, Strauss WM. Defining embryonic stem cell identity using differentiation-related microRNAs and their potential targets. Mamm Genome 2007; 18: 316-327. 
35. Papagiannakopoulos T, Kosik KS. MicroRNAs: regulators of oncogenesis and stemness. BMC Med 2008; 6: 15-18.

36. Christensen M, Schratt GM. MicroRNA involvement in developmental and functional aspects of the nervous system and in neurological diseases. Neurosci Lett 2009; in press PMID:19393715

37. Gao FB. Posttranscriptional control of neuronal development by microRNA networks Trends Neurosci 2008; 31: 20-26.

38. Kim J, Krichevsky A, Grad Y, Hayes GD, Kosik KS, Church GM et al. Drosophila microRNAs exhibit diverse spatial expression patterns during embryonic development. Proc Natl Acad Sci USA 2005; 102: 18017-18022.

39. Smirnova L, Gräfe A, Seiler A, Schumacher S, Nitsch R, Wulczyn FG. Regulation of miRNA expression during neural cell specification. Eur J Neurosci 2005; 21: 1469-1477.

40. Giraldez AJ, Cinalli RM, Glasner ME, Enright AJ, Thomson JM, Baskerville $S$ et al. MicroRNAs regulate brain morphogenesis in zebrafish. Science 2005; 308: 833-838.

41. Kim J, Inoue K, Ishii J, Vanti WB, Voronov SV, Murchison E et al. A microRNA feedback circuit in midbrain dopamine neurons. Science 2007; 317: 1220-1224

42. Schaefer A, O'Carroll D, Tan L, Hillman D, Sugimori M, Llinas R et al. Cerebellar neurodegeneration in the absence of microRNAs. J Exp Med 2007; 204: 1553-1558.

43. De Pietri Tonelli D, Pulvers JN, Haffner C, Murchison EP, Hannon GJ, Huttner WB. miRNAs are essential for survival and differentiation of newborn neurons but not for expansion of neural progenitors during early neurogenesis in the mouse embryonic neocortex. Development 2008; 135: 3911-3921.

44. Hatfield SD, Shcherbata HR, Fischer KA, Nakahara K, Carthew RW, Ruohola-Baker H. Stem cell division is regulated by the microRNA pathway. Nature 2005; 435: 974-978.

45. Gangaraju VK, Lin H. MicroRNAs: key regulators of stem cells. Nat Rev Mol Cell Biol 2009; 10: $116-125$.

46. Shi Y, Sun G, Zhao C, Stewart R. Neural stem cell self-renewal. Crit Rev Oncol Hematol 2008; 65: 43-53.

47. Krichevsky AM, Sonntag KC, Isacson O, Kosik KS. Specific microRNAs modulate embryonic stem cell-derived neurogenesis. Stem Cells 2006; 24: 857-864

48. Marson A, Levine SS, Cole MF, Frampton GM, Brambrink T, Johnstone S et al. Connecting microRNA genes to the core transcriptional regulatory circuitry of embryonic stem cells. Cell 2008; 134: 521-533.

49. Canoll P, Goldman JE. The interface between glial progenitors and gliomas. Acta Neuropathol 2008; 116: 465-477.

50. Ferretti E, De Smaele E, Po A, Di Marcotullio L, Tosi E, Espinola MS et al. MicroRNA profiling in human medulloblastoma. Int J Cancer 2009; 124: 568-577.

51. Garzia L, Andolfo I, Cusanelli E, Marino N, Petrosino G, De Martino D et al. MicroRNA199b-5p impairs cancer stem cells through negative regulation of HES1 in medulloblastoma. PLOS ONE 2009; 4: e4998.

52. Conaco C, Otto S, Han JJ, Mandel G. Reciprocal actions of REST and a microRNA promote neuronal identity. Proc Natl Acad Sci USA 2006; 103: 2422-2427.

53. Wu J, Xie X. Comparative sequence analysis reveals an intricate network among REST, CREB and miRNA in mediating neuronal gene expression. Genome Biol 2006 7: R85.

54. Makeyev EV, Zhang J, Carrasco MA, Maniatis T. The MicroRNA miR-124 promotes neuronal differentiation by triggering brain-specific alternative pre-mRNA splicing. Mol Cell 2007; 27: 435-448.

55. Visvanathan J, Lee S, Lee B, Lee JW, Lee SK. The microRNA miR-124 antagonizes the anti-neural REST/SCP1 pathway during embryonic CNS development. Genes Dev 2007; 21: $744-749$.

56. Yeo M, Lee SK, Lee B, Ruiz EC, Pfaff SL, Gill GN. Small CTD phosphatases function in silencing neuronal gene expression. Science 2005; 307: 596-600.
57. Molofsky AV, Pardal R, Iwashita T, Park IK, Clarke MF, Morrison SJ. Bmi-1 dependence distinguishes neural stem cell self-renewal from progenitor proliferation. Nature 2003; 425 962-967.

58. Sparmann A, van Lohuizen M. Polycomb silencers control cell fate, development and cancer. Nat Rev Cancer 2006; 6: 846-856.

59. Bruggeman SW, Hulsman D, Tanger E, Buckle T, Blom M, Zevenhoven J et al. Bmi controls tumor development in an Ink4a/Arf-independent manner in a mouse model for glioma. Cancer Cell 2007; 12: 328-334.

60. Zhang Y, Chao T, Li R, Liu W, Chen Y, Yan X et al. MicroRNA-128 inhibits glioma cells proliferation by targeting transcription factor E2F3a. J Mol Med 2009; 87: 43-51.

61. Varambally S, Cao Q, Mani RS, Shankar S, Wang X, Ateeq B et al. Genomic loss of microRNA-101 leads to overexpression of histone methyltransferase EZH2 in cancer Science 2008; 322: 1695-1699.

62. Sempere LF, Freemantle S, Pitha-Rowe I, Moss E, Dmitrovsky E, Ambros V. Expression profiling of mammalian microRNAs uncovers a subset of brain-expressed microRNAs with possible roles in murine and human neuronal differentiation. Genome Biol 2004; 5: R13.

63. Stark A, Brennecke J, Russell RB, Cohen SM. Identification of drosophila MicroRNA targets. PLoS Biol 2003; 1: E60.

64. Li X, Carthew RW. A microRNA mediates EGF receptor signaling and promotes photoreceptor differentiation in the Drosophila eye. Cell 2005; 123: 1267-1277.

65. Johnston Jr RJ, Chang S, Etchberger JF, Ortiz CO, Hobert O. MicroRNAs acting in a double-negative feedback loop to control a neuronal cell fate decision. Proc Natl Acad Sc USA 2005; 102: 12449-12454.

66. Tsang J, Zhu J, van Oudenaarden A. MicroRNA-mediated feedback and feedforward loops are recurrent network motifs in mammals. Mol Cell 2007; 26: 753-767.

67. Li X, Cassidy JJ, Reinke CA, Fischboeck S, Carthew RW. A microRNA imparts robustness against environmental fluctuation during development. Cell 2009; 137: 273-282.

68. Kefas B, Godlewski J, Comeau L, Li Y, Abounader R, Hawkinson M et al. microRNA-7 inhibits the epidermal growth factor receptor and the Akt pathway and is down-regulated in glioblastoma. Cancer Res 2008; 68: 3566-3572.

69. Corsten MF, Miranda R, Kasmieh R, Krichevsky AM, Weissleder R, Shah K. MicroRNA-21 knockdown disrupts glioma growth in vivo and displays synergistic cytotoxicity with neura precursor cell delivered S-TRAIL in human gliomas. Cancer Res 2007; 67: 8994-9000.

70. Papagiannakopoulos T, Shapiro A, Kosik KS. MicroRNA-21 targets a network of key tumor-suppressive pathways in glioblastoma cells. Cancer Res 2008; 68: 8164-8172.

71. Krichevsky AM, Gabriely G. miR-21: a small multi-faceted RNA. J Cell Mol Med 2009; 13 39-53.

72. Singh SK, Kagalwala MN, Parker-Thornburg J, Adams H, Majumder S. REST maintains self-renewal and pluripotency of embryonic stem cells. Nature 2008; 453: 223-227.

73. Jørgensen HF, Terry A, Beretta C, Pereira CF, Leleu M, Chen ZF et al. REST selectively represses a subset of RE1-containing neuronal genes in mouse embryonic stem cells. Development 2009; 136: 715-721.

74. Packer AN, Xing Y, Harper SQ, Jones L, Davidson BL. The bifunctional microRNA miR-9/ miR-9* regulates REST and CoREST and is downregulated in Huntington's disease. $J$ Neurosci 2008; 28: 14341-14346.

75. Shibata M, Kurokawa D, Nakao H, Ohmura T, Aizawa S. MicroRNA-9 modulates CajalRetzius cell differentiation by suppressing Foxg1 expression in mouse medial pallium. $J$ Neurosci 2008; 28: 10415-10421.

76. Nass D, Rosenwald S, Meiri E, Gilad S, Tabiban-Keissar H, Schlosberg A et al. MiR-92b and $\mathrm{miR}-9 / 9^{*}$ are specifically expressed in brain primary tumors and can be used to differentiate primary from metastatic brain tumors. Brain Pathol 2008; in press PMID:18624795. 